\title{
Superconducting orbital magnetoelectric effect and its evolution across the superconductor-normal metal phase transition
}

\author{
Wen-Yu He ${ }^{1}{ }^{1, *}$ and K. T. Law ${ }^{2, \dagger}$ \\ ${ }^{1}$ Department of Physics, Massachusetts Institute of Technology, Cambridge, Massachusetts 02139, USA \\ ${ }^{2}$ Department of Physics, Hong Kong University of Science and Technology, Clear Water Bay, Hong Kong, China
}

(Received 23 December 2020; revised 11 June 2021; accepted 15 June 2021; published 8 July 2021)

\begin{abstract}
The superconducting magnetoelectric effect, which is the current-induced magnetization in a superconductor, mainly focused on spin magnetization in previous studies, but ignored the effect of the orbital magnetic moments carried by the paired Bloch electrons. In this work, we show that orbital magnetic moments in superconductors can induce large orbital magnetization in the presence of a current. We constructed a unified description for the current-induced spin and orbital magnetization across the superconductor-normal metal phase transition. We find that in a superconductor with uniform pairing, the current-induced magnetization at a given current density is the same as that in its normal metal state, while with the nonuniform superconducting pairing, the current-induced magnetization exhibits an abrupt change in magnitude near the superconductor-normal metal phase transition. Importantly, our theory predicts the orbital magnetoelectric effect in superconducting twisted bilayer graphene which has paired Bloch electrons with large orbital magnetic moments and negligible spin-orbit coupling. We propose that the measurement of the current-induced orbital magnetoelectric effect can be used to detect the possible nonuniform pairings in twisted bilayer graphene and other newly discovered superconductors with nontrivial Berry curvatures.
\end{abstract}

DOI: 10.1103/PhysRevResearch.3.L032012

Introduction. The superconducting magnetoelectric effect is the current-induced magnetization in the superconducting state of a material. In previous studies, it mainly focused on the current-induced spin magnetization which arises from the spin-orbit coupling (SOC) in noncentrosymmetric superconductors [1-9]. Besides the SOC, nonzero Berry curvature can also arise due to the inversion symmetry breaking [10] and has an effect on the superconducting state [11]. It is known that Berry curvature introduces orbital magnetic moments to the Bloch electrons [10,12-16], and the orbital magnetic moments are the source of the current-induced orbital magnetization in a normal metal $[17,18]$. In the superconducting state, the orbital magnetic moments from the paired Bloch electrons are involved in Cooper pairs, but the effect of orbital magnetic moments has never been studied in the superconducting magnetoelectric effect. It raises the problem of how the current-induced orbital magnetization would evolve in the phase transition from the normal metal state to the superconducting state.

In this work, we show that in superconductors where the paired Bloch electrons carry orbital magnetic moments, applying current can generate orbital magnetization. Importantly, we constructed a unified description for the

\footnotetext{
*wenyuhe@mit.edu

†phlaw@ust.hk
}

Published by the American Physical Society under the terms of the Creative Commons Attribution 4.0 International license. Further distribution of this work must maintain attribution to the author(s) and the published article's title, journal citation, and DOI. current-induced spin and orbital magnetization that is applicable in both regions across the superconductor-normal metal phase transition. At the phase transition, the current-induced magnetization in the normal metal state is found to have smooth connection to that in the superconducting state when the pairing is uniform on the Fermi surfaces, while the magnetization exhibits an abrupt change in the nonuniform pairing case. The abrupt change is further ascribed to the nonuniform excitation of quasiparticles controlled by the nonuniform pairing on the Fermi surfaces.

To demonstrate the utility of our theory, we study the case of twisted bilayer graphene (TBG) and predict that the TBG exhibits current-induced orbital magnetization in the superconductivity region. The TBG has recently been observed to exhibit both superconductivity [19-24] and orbital magnetism [24-28]. Importantly, the superconductivity and orbital magnetism have been observed in the same TBG sample (at different filling factors) [24], indicating that the Cooper pairs are formed by electrons carrying finite orbital magnetic moments. Here we find that the orbital magnetic moments from the paired Bloch electrons can give rise to current-induced orbital magnetization in superconducting TBG. More importantly, we point out that the measurement of current-induced orbital magnetization across the superconductor-normal metal phase transition in TBG can test whether superconducting TBG has uniform or nonuniform pairing order parameters. Besides being applicable to TBG, our theory is generally applicable to a large number of noncentrosymmetric superconductors with finite Berry curvatures.

A unified description for the magnetoelectric effect. Both the normal and superconducting states of a material can be 
described by the Bogoliubov-de Gennes Hamiltonian [29]

$$
\mathcal{H}=\frac{1}{2} \sum_{\boldsymbol{k}}\left(\begin{array}{cc}
c_{k}^{\dagger} & \left.c_{-\boldsymbol{k}}\right)
\end{array}\right)\left(\begin{array}{cc}
H_{0}(\boldsymbol{k}) & \hat{\Delta}(\boldsymbol{k}) \\
\hat{\Delta}^{\dagger}(\boldsymbol{k}) & -H_{0}^{*}(-\boldsymbol{k})
\end{array}\right)\left(\begin{array}{c}
c_{\boldsymbol{k}} \\
c_{-\boldsymbol{k}}^{\dagger}
\end{array}\right) .
$$

Here $H_{0}(\boldsymbol{k})$ is the normal state Hamiltonian matrix, $\hat{\Delta}(\boldsymbol{k})$ is the pairing matrix, and $c_{k}^{\dagger}\left(c_{k}\right)$ is the creation (annihilation) operator that includes multiple components for all the orbital and spin degrees of the system. In the normal state, a Bloch electronic state $\left|\phi_{v, \boldsymbol{k}}\right\rangle$ with energy $\xi_{v, \boldsymbol{k}}=\left\langle\phi_{v, \boldsymbol{k}}\left|H_{0}(\boldsymbol{k})\right| \phi_{v, \boldsymbol{k}}\right\rangle$ carries the spin magnetic moment $\boldsymbol{S}_{v, k}=\left\langle\phi_{v, k}\left|\frac{1}{2} \mu_{\mathrm{B}} g \sigma\right| \phi_{v, k}\right\rangle$, with $\mu_{\mathrm{B}}$ being the Bohr magneton and $g$ the Landé $g$ factor. The SOC in the material is manifested by the locking of the spin magnetic moment direction with the group velocity $\boldsymbol{v}_{v, k}=$ $\partial_{\boldsymbol{k}} \xi_{v, \boldsymbol{k}}$. In the absence of inversion symmetry, nonzero Berry curvature $\boldsymbol{\Omega}_{v, \boldsymbol{k}}=i\left\langle\partial_{\boldsymbol{k}} \phi_{v, \boldsymbol{k}}|\times| \partial_{\boldsymbol{k}} \phi_{v, \boldsymbol{k}}\right\rangle$ can arise and endow the Bloch electronic state with a finite orbital magnetic moment $\boldsymbol{m}_{v, \boldsymbol{k}}=\frac{i e}{2 \hbar}\left\langle\partial_{\boldsymbol{k}} \phi_{v, \boldsymbol{k}}\left|\times\left[H_{0}(\boldsymbol{k})-\xi_{v, \boldsymbol{k}}\right]\right| \partial_{\boldsymbol{k}} \boldsymbol{\phi}_{v, \boldsymbol{k}}\right\rangle[10,12,13]$. Therefore, the total magnetic moment of a Bloch electron is $\boldsymbol{M}_{v, \boldsymbol{k}}=\boldsymbol{S}_{v, \boldsymbol{k}}+\boldsymbol{m}_{v, \boldsymbol{k}}$. In the superconducting state with timereversal symmetry, as a Bloch electronic state $\left|\phi_{\nu, k}\right\rangle$ always has a partner $\left|\phi_{\nu,-k}\right\rangle$ with the same energy $\xi_{v, k}=\xi_{v,-k}$, the two Bloch electronic states can pair and give rise to the pairing order parameter $\Delta_{v, \boldsymbol{k}} \sim\left\langle\phi_{\nu,-\boldsymbol{k}} \phi_{v, \boldsymbol{k}}\right\rangle$ [29,30], yielding the Bogoliubov quasiparticle spectrum $\epsilon_{v, k}=\sqrt{\xi_{v, k}^{2}+\left|\Delta_{v, k}\right|^{2}}$. Therefore, in a noncentrosymmetric superconductor with finite Berry curvature, the paired electrons generally carry both spin and orbital magnetic moments even though the net magnetization is zero in the absence of a current. However, as we show below, applying a current can induce net spin and orbital magnetization.

Applying a current to a metal or superconductor can be described by introducing a $U(1)$ gauge field to the original Hamiltonian $\mathcal{H} \rightarrow \mathcal{H}+\delta \mathcal{H}$ where

$$
\begin{aligned}
\delta \mathcal{H}= & -\sum_{\boldsymbol{k}, \boldsymbol{q}} c_{\boldsymbol{k}-\frac{1}{2} \boldsymbol{q}}^{\dagger} c_{\boldsymbol{k}+\frac{1}{2} \boldsymbol{q}}\left[\frac{e}{\hbar} \partial_{k_{i}} H_{0}(\boldsymbol{k}) A_{i}(-\boldsymbol{q}, t)\right. \\
& \left.-\frac{e^{2}}{2 \hbar^{2}} \partial_{k_{i} k_{j}}^{2} H_{0}(\boldsymbol{k}) A_{i}(\boldsymbol{q}, t) A_{j}(-\boldsymbol{q}, t)\right],
\end{aligned}
$$

with the spatial components denoted by $i, j=x, y, z$, and $\boldsymbol{A}(\boldsymbol{r}, t)=\sum_{q} \boldsymbol{A}(\boldsymbol{q}, t) e^{i \boldsymbol{q} \cdot \boldsymbol{r}}$ being the vector gauge potential. The perturbation $\delta \mathcal{H}$ changes the distribution of Bloch electrons at the Fermi energy, and also changes the original Fermi surfaces, as is shown in Fig. 1(a). In the normal metallic state, the redistribution of Bloch electrons results in an imbalance of the total magnetic moments of the occupied states, so a net magnetization arises by applying a current. In the superconducting state, the Bloch electrons on the Fermi surfaces with net momentum $\boldsymbol{q}$ are paired into Cooper pairs, which have the form $\Delta_{v, k, \boldsymbol{q}} \sim\left\langle\phi_{v,-\boldsymbol{k}+\frac{1}{2} \boldsymbol{q}} \phi_{v, \boldsymbol{k}+\frac{1}{2} \boldsymbol{q}}\right\rangle$. The Cooper pairs with net momentum generate a supercurrent, and also induce net magnetization that comes from the spin and orbital magnetic moments in the paired Bloch electrons. Besides the Cooper pairs, quasiparticles with net momentum are excited at finite temperature and also contribute to the net magnetization. Therefore the net magnetization in a superconductor comes from two aspects: One is the supercurrent and the other is the quasiparticle current, as is schematically illustrated in
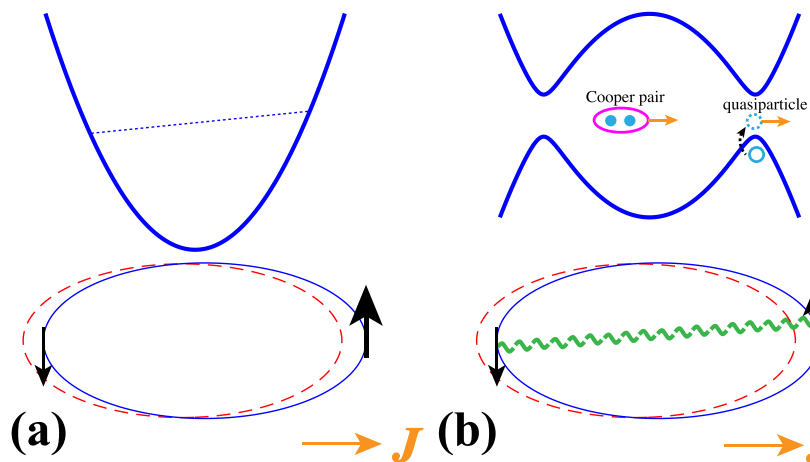

FIG. 1. Applying a current to a normal metal (a) and a superconductor (b). The blue and red circles correspond to the Fermi surface with and without current, respectively. In a normal metal, applying a current makes more electronic states with momentum parallel to the applied current density $\boldsymbol{J}$ occupied at the Fermi energy. The occupation is schematically illustrated by the blue dotted line in (a). As Bloch electrons carry magnetic moments, the redistribution at the Fermi energy gives rise to net magnetization. In a superconductor, the Bloch electrons of net momentum get paired to form Cooper pairs of net momentum. The magnetic moments carried by the paired Bloch electrons are involved in the pairing condensation and can generate a net magnetization. At finite temperature, there are quasiparticle excitations in the superconducting state, so the excited quasiparticles also contribute to the current and current-induced magnetization at $0<T<T_{\mathrm{c}}$. The black arrow denotes the total magnetic moments carried by the electrons moving forward or backward, and the amplitude of the magnetic moment is schematically represented by the size of the arrow.

Fig. 1(b). Importantly, the way for a supercurrent to induce net magnetization is in analogy to that in the normal metallic state: in the superconductivity the supercurrent accumulates net spin and orbital magnetic moments in the pairing condensation, while in the normal metal the current populates net magnetic moments at the Fermi energy.

To calculate the current-induced magnetization in both the normal and the superconducting states, we apply the linear response theory and obtain the current-induced bulk magnetization as [29]

$$
\begin{aligned}
M_{i}= & -\frac{e}{\hbar} \sum_{\nu} \int_{\boldsymbol{k}} M_{\nu, \boldsymbol{k}, i} v_{v, \boldsymbol{k}, j}\left\{\frac{\tau}{1-i \omega \tau} \frac{d f\left(\epsilon_{v, \boldsymbol{k}}\right)}{d \epsilon_{v, \boldsymbol{k}}} E_{j}\right. \\
& \left.+\left[\frac{d f\left(\epsilon_{v, \boldsymbol{k}}\right)}{d \epsilon_{v, \boldsymbol{k}}}-\frac{d f\left(\xi_{v, \boldsymbol{k}}\right)}{d \xi_{v, \boldsymbol{k}}}\right] A_{j}\right\} .
\end{aligned}
$$

This magnetization is associated with the current density

$$
\begin{aligned}
J_{i}= & -\frac{e^{2}}{\hbar^{2}} \sum_{v} \int_{\boldsymbol{k}} v_{v, \boldsymbol{k}, i} v_{v, \boldsymbol{k}, j}\left\{\frac{\tau}{1-i \omega \tau} \frac{d f\left(\epsilon_{v, \boldsymbol{k}}\right)}{d \epsilon_{v, \boldsymbol{k}}} E_{j}\right. \\
& \left.+\left[\frac{d f\left(\epsilon_{v, \boldsymbol{k}}\right)}{d \epsilon_{v, \boldsymbol{k}}}-\frac{d f\left(\xi_{v, \boldsymbol{k}}\right)}{d \xi_{v, \boldsymbol{k}}}\right] A_{j}\right\} .
\end{aligned}
$$

Here, $\int_{\boldsymbol{k}} \equiv \int_{\mathrm{BZ}} d \boldsymbol{k} /(2 \pi)^{d}$ with $d$ being the dimension, $\tau$ is the effective scattering time, and $f(\epsilon)$ is the Fermi Dirac distribution function. In the derivation of Eqs. (3) and (4) we applied the Coulomb gauge so the electric field takes the form $\boldsymbol{E}=i \omega \boldsymbol{A}$ [31]. The bulk magnetization derived in Eq. (3) involves both the spin and orbital magnetization, and 
it is applicable in both the normal metal and the superconductivity region. In the normal metal region with zero pairing gap $\Delta_{v, k}=0$, Eq. (4) is the standard Drude formula to describe the current density under an applied electric field $\boldsymbol{E}$, and the current-induced magnetization in Eq. (3) recovers the magnetoelectric susceptibility of a metal $[17,18]$. In the superconductivity region with finite pairing gap $\left|\Delta_{v, k}\right|$, the first terms in Eqs. (3) and (4) describe the net magnetization and the associated current density from the excited quasiparticles. As the quasiparticle excitations are suppressed by the pairing gap, the current and magnetization that come from the quasiparticles approach to zero at low temperature. The second terms in Eqs. (3) and (4) describe the magnetization and supercurrent density from Cooper pairs. The supercurrent in Eq. (4) arises from the vector gauge field $\boldsymbol{A}$, which twists the phase of pairing condensation. The second term in Eq. (3) is the net magnetization of pairing condensation induced by the supercurrent. Importantly, as the temperature decreases, the supercurrent and the supercurrent-induced magnetization become dominant.

For the current-induced magnetization described in Eqs. (3) and (4), the vector fields $\boldsymbol{E}$ and $\boldsymbol{A}$ can further be replaced by the current density $\boldsymbol{J}$, which results in the following expression:

$$
M_{i}=\alpha_{i k} J_{k} \quad \text { with } \quad \alpha_{i k}=\gamma_{i j}\left(\tilde{v}^{-1}\right)_{j k} .
$$

Here the susceptibility tensor $\alpha_{i j}$ describes the magnetization induced by a unit current density and can be nonzero only for crystals with point group symmetry belonging to one of the 18 gyrotropic point groups [9,32]. The tensors $\gamma_{i j}, \tilde{v}_{j k}$ that are used to calculate $\alpha_{i j}$ are

$$
\begin{aligned}
& \gamma_{i j}=\frac{e}{\hbar} \sum_{\nu} \int_{\boldsymbol{k}} M_{\nu, \boldsymbol{k}, i} v_{v, \boldsymbol{k}, j}\left[\frac{d f\left(\xi_{v, \boldsymbol{k}}\right)}{d \xi_{v, \boldsymbol{k}}}-\frac{1}{1-i \omega \tau} \frac{d f\left(\epsilon_{v, \boldsymbol{k}}\right)}{d \epsilon_{v, \boldsymbol{k}}}\right], \\
& \tilde{v}_{j k}=\frac{e^{2}}{\hbar^{2}} \sum_{\nu} \int_{\boldsymbol{k}} v_{v, \boldsymbol{k}, j} v_{v, \boldsymbol{k}, k}\left[\frac{d f\left(\xi_{v, \boldsymbol{k}}\right)}{d \xi_{v, \boldsymbol{k}}}-\frac{1}{1-i \omega \tau} \frac{d f\left(\epsilon_{v, \boldsymbol{k}}\right)}{d \epsilon_{v, \boldsymbol{k}}}\right] .
\end{aligned}
$$

Importantly, at zero temperature $T=0 \quad \mathrm{~K}$, it is found that $\gamma_{i j} \sim \frac{e}{\hbar} \sum_{\nu} \oint M_{\nu, \boldsymbol{k}_{\mathrm{F}}, i} v_{v, \boldsymbol{k}_{\mathrm{F}}, j} d \boldsymbol{k}_{\mathrm{F}}$ and $\tilde{v}_{i j} \sim$ $\frac{e^{2}}{\hbar^{2}} \sum_{v} \oint v_{v, \boldsymbol{k}_{\mathrm{F}}, i} v_{v, \boldsymbol{k}_{\mathrm{F}}, j} d \boldsymbol{k}_{\mathrm{F}}$ [29], with $\boldsymbol{k}_{\mathrm{F}}$ being the wave vector on the Fermi surfaces, so the induced magnetization $\boldsymbol{M}$ at a given current density $\boldsymbol{J}$ at $T=0 \mathrm{~K}$ is the same regardless of whether the system is superconducting or not. The susceptibility $\alpha_{i j}$ at $T=0 \mathrm{~K}$ is only determined by the group velocity and total magnetic moments of Bloch electrons on the Fermi surfaces. It indicates that for a normal metal that has current-induced orbital magnetization, the orbital magnetization can also arise from applying supercurrent in its superconductivity region. In the temperature range $0<T<T_{\mathrm{c}}$ ( $T_{\mathrm{c}}$ is the critical temperature for a superconductor), as the excited quasiparticles come into play a role in the current-induced magnetization, the pairing gap that controls the quasiparticle excitations becomes another factor that can affect the magnetization at given current density.

Nonuniform pairing induced abrupt change in the currentinduced magnetization. The effect of quasiparticle excitations on the current-induced magnetization is the most prominent at the temperature near the superconductor-normal metal phase transition, where the small pairing gap near $T_{\mathrm{c}}$ allows a large number of quasiparticle excitations to coexist with Cooper pairs. From the first term in Eqs. (3) and (4) it is known that the current from the excited quasiparticles and the affiliated magnetization are inherited from those in the normal metal state. In the case of uniform pairing gap $\left|\Delta_{\nu, \boldsymbol{k}_{\mathrm{F}}}\right|=\Delta_{0}$, since the suppression of quasiparticle excitations is uniform on the Fermi surfaces, the quasiparticle current and the affiliated magnetization are produced in the same ratio as the current and the current-induced magnetization in the normal metal state. In contrast, when the pairing gap $\Delta_{v, \boldsymbol{k}_{\mathrm{F}}}$ is nonuniform on Fermi surfaces, the ratio of quasiparticle current and the affiliated magnetization differs from that in the normal metal. We know from the $T=0 \mathrm{~K}$ result that the ratio of the supercurrent and the supercurrent-induced magnetization is the same as the ratio in the normal metal state. Therefore, across the superconductor-normal metal phase transition, the currentinduced magnetization is exactly the same in the uniform pairing case, while an abrupt change in magnitude can happen in the nonuniform pairing case.

The abrupt change of current-induced magnetization at $T_{\mathrm{c}}$ in the nonuniform pairing case can be deduced from the Taylor expansion of $\gamma_{i j}, \tilde{v}_{i j}$ in terms of $\Delta_{v, \boldsymbol{k}}$, which yields $\gamma_{i j} \sim \frac{e}{\hbar} \sum_{\nu} \oint M_{v, \boldsymbol{k}_{\mathrm{F}}} v_{v, \boldsymbol{k}_{\mathrm{F}}, j}\left|\Delta_{v, \boldsymbol{k}_{\mathrm{F}}}\right|^{2} d \boldsymbol{k}_{\mathrm{F}}$ and $\quad \tilde{v}_{i j} \sim \frac{e^{2}}{\hbar^{2}} \sum_{v} \oint v_{v, \boldsymbol{k}_{\mathrm{F}}, i} v_{v, \boldsymbol{k}_{\mathrm{F}}, j}\left|\Delta_{v, \boldsymbol{k}_{\mathrm{F}}}\right|^{2} d \boldsymbol{k}_{\mathrm{F}} \quad$ [29]. Above $T_{\mathrm{c}}$, we know $\gamma_{i j} \sim \frac{e}{\hbar} \sum_{\nu} \oint M_{v, \boldsymbol{k}_{\mathrm{F}}, i} v_{v, \boldsymbol{k}_{\mathrm{F}}, j} d \boldsymbol{k}_{\mathrm{F}}$ and $\tilde{v}_{i j} \sim$ $\frac{e^{2}}{\hbar^{2}} \sum_{\nu} \oint v_{v, \boldsymbol{k}_{\mathrm{F}}, i} v_{v, \boldsymbol{k}_{\mathrm{F}}, j} d \boldsymbol{k}_{\mathrm{F}}$ [29], so the dependence on the paring $\Delta_{v, k_{\mathrm{F}}}$ arises abruptly in the susceptibility $\alpha_{i j}$ once $T<T_{\mathrm{c}}$. When the pairing is nonuniform on the Fermi surfaces, the susceptibility $\alpha_{i j}$ thus differs at the two sides of $T_{\mathrm{c}}$. Such abrupt change of current-induced magnetization across $T_{\mathrm{c}}$ is an indicator of the nonuniform pairing in the superconducting state.

The orbital magnetoelectric effect in superconducting twisted bilayer graphene. In previous studies, current-induced magnetization in the superconducting state only involves the spin magnetization caused by SOC [1-9]. Recently, superconductivity was observed in TBG which has negligibly small SOC. Here, we predict that a large orbital magnetization can be induced by a current in superconducting TBG. More importantly, the behavior of the current-induced orbital magnetization in TBG near $T_{\mathrm{c}}$ provides further information about the pairing gap in TBG.

The TBG that shows superconductivity in experiment lies on the hBN substrate, so the coupling of the bottom graphene layer with the hBN can gap out the Dirac points and generate finite Berry curvature [33-36]. Due to the mismatch between the $\mathrm{hBN}$ and the bottom graphene layer, the equilateral triangle moiré superlattice has been observed to get deformed [37-39], indicating that the $C_{3}$ symmetry of the TBG is generally broken by the strain from the hBN substrate. The resulting TBG system has the lowest $C_{1}$ symmetry with finite Berry curvature, so an in-plane current can give rise to an out-of-plane orbital magnetization $M_{z}=\alpha_{z x} J_{x}+\alpha_{z y} J_{y}$ in the normal state [40]. Since at the $T=0 \mathrm{~K}$ the current-induced magnetization in the normal state is the same as the supercurrent-induced magnetization, the current-induced orbital magnetization in 


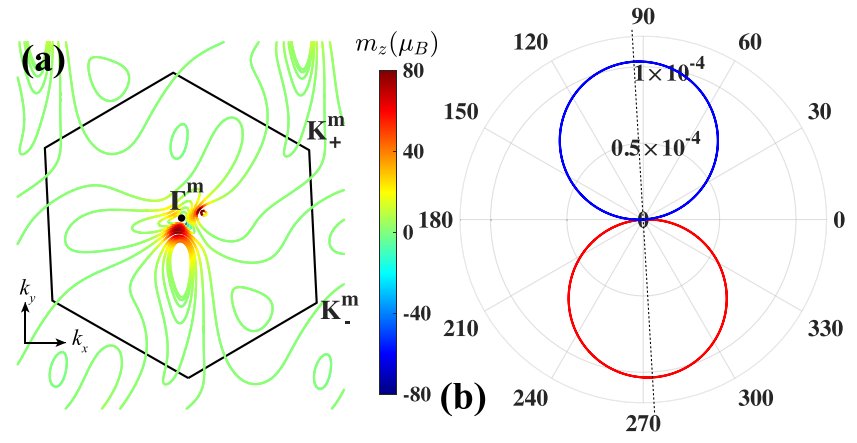

FIG. 2. (a) The orbital magnetic moments at different energy contours of the valence band. The tilted hexagon is the mini-Brillouin zone. Here orbital magnetic moment distribution in one valley is shown, and it can be mapped to the case in the other valley through time-reversal symmetry. (b) The out-of-plane orbital magnetization $M_{z}$ (in units of $\mu_{\mathrm{B}} / \mathrm{nm}^{2}$ ) induced by in-plane current of $1 \mathrm{nA} / \mathrm{nm}$ along different directions. The optimal direction that generates the largest $M_{z}$ is labeled by a black dashed line. The red and blue lines mean positive and negative values of orbital magnetization $M_{z}$, respectively. The $x$ direction has been defined to be along the angular bisector between the two zigzag directions of the top and bottom graphene layers.

TBG is always maintained in its superconducting state. To demonstrate the in-plane current-induced out-of-plane orbital magnetization in a superconducting TBG on $\mathrm{hBN}$, we construct the continuum model for a TBG with a twist angle of $1.6^{\circ}$ [41-43]. In the TBG, there is a uniaxial strain applied along the zigzag direction in the bottom graphene layer to have it stretched by $0.1 \%$ (the detailed description for the strained TBG can be found in the Supplemental Material [29]). With the massive Dirac gap of $34 \mathrm{meV}[44,45]$, the Bloch electrons in the valence moiré flat band carry orbital magnetic moments up to $80 \mu_{\mathrm{B}}$ as shown in Fig. 2(a). Around half filling in the valence moiré flat band, the in-plane supercurrent-induced out-of-plane orbital magnetization at $T=0 \mathrm{~K}$ is directly computed through Eqs. (6) and (7). Assuming the in-plane supercurrent density to be $1 \mathrm{nA} / \mathrm{nm}$, the out-of-plane orbital magnetization as a function of the inplane supercurrent direction is shown in Fig. 2(b). The largest orbital magnetization induced by the in-plane current along the optimal direction reaches the order of $10^{-4} \mu_{\mathrm{B}} / \mathrm{nm}^{2}$. This magnitude of orbital magnetization is comparable to the current-induced spin polarization in large Rashba SOC materials under $E=10^{3} \sim 10^{4} \mathrm{~V} / \mathrm{m}$, such as $\mathrm{Au}$ (111) surfaces and $\mathrm{Bi} / \mathrm{Ag}$ bilayers [46,47].

Near the superconductor-normal metal phase transition, the proportion of orbital magnetization that comes from the quasiparticle current becomes considerable, so the pairing gap that controls the quasiparticle excitations will affect the current-induced orbital magnetization near $T_{\mathrm{c}}$. For the superconducting TBG on $\mathrm{hBN}$, a possible nonuniform singlet pairing can be approximated as $\Delta_{v, k} / \Delta_{0}=1+\lambda\{\cos (\boldsymbol{k}$. $\left.\left.\tilde{\boldsymbol{a}}_{1}\right)+\cos \left(\boldsymbol{k} \cdot \tilde{\boldsymbol{a}}_{2}\right)+\cos \left[\boldsymbol{k} \cdot\left(\tilde{\boldsymbol{a}}_{1}-\tilde{\boldsymbol{a}}_{2}\right)\right]\right\} \quad[48-52]$, with $\Delta_{0}=$ $1.76 k_{\mathrm{B}} T_{\mathrm{c}} \tanh \left(1.78 \sqrt{T_{\mathrm{c}} / T-1}\right)$ and $\tilde{\boldsymbol{a}}_{1}, \tilde{\boldsymbol{a}}_{2}$ being the primitive lattice vectors of the moiré superlattice under strain [29]. The nonuniform pairing gap at $\lambda=0.5$ is plotted in the

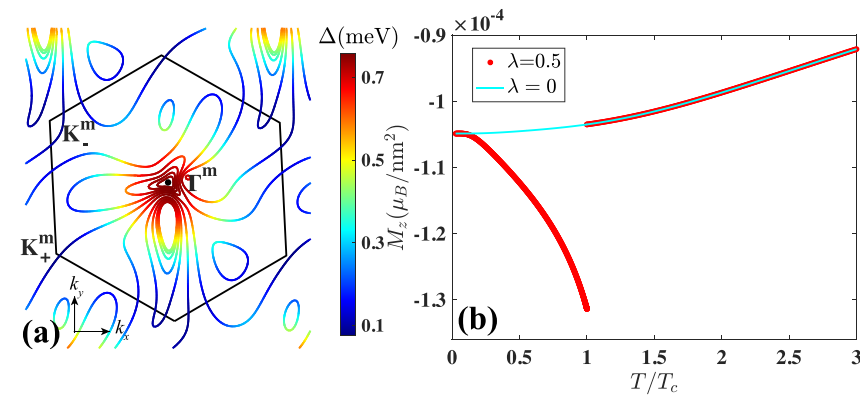

FIG. 3. (a) The pairing gap at different energy contours of the valence band. The pairing is plotted in one valley and that in the other valley can be obtained through time-reversal symmetry. (b) The current-induced out-of-plane orbital magnetization across the superconductor-normal metal phase transition. The applied in-plane current density is $1 \mathrm{nA} / \mathrm{nm}$ and the direction is along the optimal direction labeled in Fig. 2(b). The out-of-plane orbital magnetization exhibits an abrupt jump in the nonuniform pairing case while it shows smooth transition across $T_{\mathrm{c}}$ in the uniform paring case.

mini-Brillouin-zone shown in Fig. 3(a), and the orbital magnetization induced by the current along the optimal direction clearly exhibits an abrupt jump at $T_{\mathrm{c}}$ as seen in Fig. 3(b). In contrast, in the uniform pairing case which has $\lambda=0$, the current-induced orbital magnetization has smooth connection across $T_{\mathrm{c}}$. This is consistent with our analysis that an abrupt change of the current-induced magnetization at $T_{\mathrm{c}}$ indicates the pairing is nonuniform on the Fermi surfaces.

Discussion. Experimentally, the magnetization that involves orbital magnetic moments' polarization in the normal state of materials has been observed through the optical Kerr effect [53], nuclear magnetic resonance measurements [54], and the superconducting quantum interference device (SQUID) [28], and these techniques can all be used to detect the magnetization in the superconducting state [55-57]. In the recent direct image of orbital magnetism in TBG [28], it can be deduced from the measurement that the surface magnetism at the order of $10^{-4} \mu_{\mathrm{B}} / \mathrm{nm}^{2}$ corresponds to the generated static magnetic field around $1 \mathrm{nT}$, so a SQUID device with a resolution of $0.1 \mathrm{nT}$ will be able to detect the orbital magnetization induced by the current density at $1 \mathrm{nA} / \mathrm{nm}$ in superconducting TBG. The current-induced orbital magnetization and the possible abrupt change at $T_{\mathrm{c}}$ is proportional to the applied current density in the material, so the critical current density in the superconducting state sets the upper limit of the current-induced orbital magnetization below $T_{\mathrm{c}}$. As a result, superconductors with larger pairing gap that have larger critical current density would be preferred to make the effect more measurable.

It is important to note that our theory applies to a large number of recently discovered superconductors with Berry curvatures and negligible SOC, such as a trilayer graphene moiré superlattice on hBN [58-61], bilayer graphene/hBN superlattices [62], and twisted double bilayer graphene $[63,64]$. Moreover, our theory also applies to noncentrosymmetric superconductors with strong SOC and finite Berry curvatures such as chiral crystals, $\mathrm{Li}_{2} \mathrm{Pt}_{3} \mathrm{~B}$ [65], $\mathrm{Li}_{2} \mathrm{Pd}_{3} \mathrm{~B}$ [66], $\mathrm{Mo}_{3} \mathrm{Al}_{2} \mathrm{C}$ [67], and $\mathrm{TaRh}_{2} \mathrm{~B}_{2}$ and $\mathrm{NbRh}_{2} \mathrm{~B}_{2}$ [68], which were recently discovered to have superconductivity. In these chiral 
crystals the orbital magnetic moment carried by the Bloch electrons originates from the Weyl SOC [69], so the spin and orbital magnetization are strongly mixed and the orbital magnetization effect cannot be ignored.
Acknowledgment. The authors would like to thank K. F. Mak for inspiring discussions. K.T.L. acknowledges the support of the Croucher Foundation and HKRGC through Grants No. C6025-19G, No. 16310219, and No. 16309718.
[1] L. S. Levitov, Yu. V. Nazarov, and G. M. Eliashberg, Magnetostatics of superconductors without an inversion center, JETP Lett. 41, 445 (1985).

[2] V. M. Edelstein, Characteristics of the Cooper pairing in twodimensional noncentrosymmetric electron systems, Sov. Phys. JETP 68, 1244 (1989).

[3] V. M. Edelstein, Magnetoelectric Effect in Polar Superconductors, Phys. Rev. Lett. 75, 2004 (1995).

[4] S. K. Yip, Two-dimensional superconductivity with strong spinorbit interaction, Phys. Rev. B 65, 144508 (2002).

[5] K. V. Samokhin, Magnetic properties of superconductors with strong spin-orbit coupling, Phys. Rev. B 70, 104521 (2004).

[6] S. Fujimoto, Magnetoelectric effects in heavy-fermion superconductors without inversion symmetry, Phys. Rev. B 72, 024515 (2005).

[7] E. Bauer and M. Sigrist, Noncentrosymmetric Superconductors (Springer, Berlin, 2012).

[8] G. Tkachov, Magnetoelectric Andreev Effect due to ProximityInduced Nonunitary Triplet Superconductivity in Helical Metals, Phys. Rev. Lett. 118, 016802 (2017).

[9] W.-Y. He and K. T. Law, Magnetoelectric effects in gyrotropic superconductors, Phys. Rev. Research 2, 012073(R) (2020).

[10] D. Xiao, M.-C. Chang, and Q. Niu, Berry phase effects on electronic properties, Rev. Mod. Phys. 82, 1959 (2010).

[11] Z. Wang, L. Dong, C. Xiao, and Q. Niu, Berry Curvature Effects on Quasiparticle Dynamics in Superconductors, Phys. Rev. Lett. 126, 187001 (2021).

[12] M.-C. Chang and Q. Niu, Berry phase, hyperorbits, and the Hofstadter spectrum: Semiclassical dynamics in magnetic Bloch bands, Phys. Rev. B 53, 7010 (1996).

[13] G. Sundaram and Q. Niu, Wave-packet dynamics in slowly perturbed crystals: Gradient corrections and Berry-phase effects, Phys. Rev. B 59, 14915 (1999).

[14] D. Xiao, J. Shi, and Q. Niu, Berry Phase Correction to Electron Density of States in Solids, Phys. Rev. Lett. 95, 137204 (2005).

[15] T. Thonhauser, D. Ceresoli, D. Vanderbilt, and R. Resta, Orbital Magnetization in Periodic Insulators, Phys. Rev. Lett. 95, 137205 (2005).

[16] D. Ceresoli, T. Thonhauser, D. Vanderbilt, and R. Resta, Orbital magnetization in crystalline solids: Multi-band insulators, Chern insulators, and metals, Phys. Rev. B 74, 024408 (2006).

[17] S. Zhong, J. E. Moore, and I. Souza, Gyrotropic Magnetic Effect and the Magnetic Moment on the Fermi Surface, Phys. Rev. Lett. 116, 077201 (2016).

[18] J. Ma and D. A. Pesin, Chiral magnetic effect and natural optical activity in metals with or without Weyl points, Phys. Rev. B 92, 235205 (2015).

[19] Y. Cao et al., Unconventional superconductivity in magic-angle graphene superlattices, Nature (London) 556, 43 (2018).

[20] M. Yankowitz et al., Tuning superconductivity in twisted bilayer graphene, Science 363, 1059 (2019).
[21] E. Codecido et al., Correlated insulating and superconducting states in twisted bilayer graphene below the magic angle, Sci. Adv. 5, eaaw9770 (2019).

[22] Y. Saito, J. Ge, K. Watanabe, T. Taniguchi, and A. F. Young, Independent superconductors and correlated insulators in twisted bilayer graphene, Nat. Phys. 16, 926 (2020).

[23] P. Stepanov et al., Untying the insulating and superconducting orders in magic-angle graphene, Nature (London) 583, 375 (2020).

[24] X. Lu et al., Superconductors, orbital magnets and correlated states in magic-angle bilayer graphene, Nature (London) $\mathbf{5 7 4}$ 653 (2019).

[25] A. L. Sharpe et al., Emergent ferromagnetism near threequarters filling in twisted bilayer graphene, Science 365, 605 (2019).

[26] M. Serlin et al., Intrinsic quantized anomalous Hall effect in a moiré heterostructure, Science 367, 900 (2019).

[27] H. Polshyn, J. Zhu, M. A. Kumar, Y. Zhang, F. Yang, C. L. Tschirhart, M. Serlin, K. Watanabe, T. Taniguchi, A. H MacDonald, and A. F. Young, Electrical switching of magnetic order in an orbital Chern insulator, Nature (London) 588, 66 (2020).

[28] C. L. Tschirhart et al., Imaging orbital ferromagnetism in a moiré Chern insulator, Science 372, 1323 (2021).

[29] See Supplemental Material at http://link.aps.org/supplemental/ 10.1103/PhysRevResearch.3.L032012 for (i) the details about the Bogoliubov-de Gennes Hamiltonian in the eigenbasis, (ii) the detailed derivation of the current-induced magnetization in both the normal metal and superconductivity region, and (iii) the continuum model of twisted bilayer graphene on boron nitride with uniaxial strain.

[30] A. Ramires and M. Sigrist, Identifying detrimental effects for multiorbital superconductivity: Application to $\mathrm{Sr}_{2} \mathrm{RuO}_{4}$, Phys. Rev. B 94, 104501 (2016).

[31] J. R. Schrieffer, Theory of Superconductivity, Advanced Book Program (Avalon Publishing, 1999).

[32] F. de Juan, A. G. Grushin, T. Morimoto, and J. E. Moore, Quantized circular photogalvanic effect in Weyl semimetals, Nat. Commun. 8, 15995 (2017).

[33] M. Xie and A. H. MacDonald, Nature of the Correlated Insulator States in Twisted Bilayer Graphene, Phys. Rev. Lett. 124, 097601 (2020).

[34] Y.-H. Zhang, D. Mao, and T. Senthil, Twisted bilayer graphene aligned with hexagonal boron nitride: Anomalous Hall effect and a lattice model, Phys. Rev. Research 1, 033126 (2019).

[35] Y.-H. Zhang, D. Mao, Y. Cao, P. Jarillo.-Herrero, and T. Senthil, Nearly flat Chern bands in moiré superlattices, Phys. Rev. B 99 075127 (2019).

[36] N. Bultinck, S. Chatterjee, and M. P. Zaletel, Mechanism for Anomalous Hall Ferromagnetism in Twisted Bilayer Graphene, Phys. Rev. Lett. 124, 166601 (2020). 
[37] Y. Choi et al., Electronic correlations in twisted bilayer graphene near the magic angle, Nat. Phys. 15, 1174 (2019).

[38] A. Kerelsky et al., Maximized electron interactions at the magic angle in twisted bilayer graphene, Nature (London) 572, 95 (2019).

[39] Y. Xie et al., Spectroscopic signatures of many-body correlations in magic-angle twisted bilayer graphene, Nature (London) 572, 101 (2019).

[40] W.-Y. He, D. G. Gordon, and K. T. Law, Giant orbital magnetoelectric effect and current-induced magnetization switching in twisted bilayer graphene, Nat. Commun. 11, 1650 (2020).

[41] J. M. B. L. Santos, N. M. R. Peres, and A. H. C. Neto, Graphene Bilayer with a Twist: Electronic Structure, Phys. Rev. Lett. 99, 256802 (2007).

[42] J. M. B. L. Santos, N. M. R. Peres, and A. H. C. Neto, Continuum model of the twisted graphene bilayer, Phys. Rev. B 86, 155449 (2012).

[43] R. Bistritzer and A. H. MacDonald, Moiré bands in twisted double-layer graphene, Proc. Natl. Acad. Sci. USA 108, 12233 (2011).

[44] M. Lee et al., Ballistic miniband conduction in a graphene superlattice, Science 353, 1526 (2016).

[45] H. Kim et al., Accurate gap determination in monolayer and bilayer graphene/h-BN moiré superlattices, Nano Lett. 18, 7732 (2018).

[46] A. Johansson, J. Henk, and I. Mertig, Theoretical aspects of the Edelstein effect for anisotropic two-dimensional electron gas and topological insulators, Phys. Rev. B 93, 195440 (2016).

[47] A. Johansson, J. Henk, and I. Mertig, Edelstein effect in Weyl semimetals, Phys. Rev. B 97, 085417 (2018).

[48] B. Lian, Z. Wang, and B. A. Bernevig, Twisted Bilayer Graphene: A Phonon-Driven Superconductor, Phys. Rev. Lett. 122, 257002 (2019).

[49] F. Wu and S. Das Sarma, Identification of superconducting pairing symmetry in twisted bilayer graphene using in-plane magnetic field and strain, Phys. Rev. B 99, 220507(R) (2019).

[50] F. Wu, A. H. MacDonald, and I. Martin, Theory of PhononMediated Superconductivity in Twisted Bilayer Graphene, Phys. Rev. Lett. 121, 257001 (2018).

[51] F. Wu, Topological chiral superconductivity with spontaneous vortices and supercurrent in twisted bilayer graphene, Phys. Rev. B 99, 195114 (2019).

[52] T. J. Peltonen, R. Ojajarvi, and T. T. Heikkila, Mean-field theory for superconductivity in twisted bilayer graphene, Phys. Rev. B 98, 220504(R) (2018).

[53] J. Lee, Z. Wang, H. Xie, K. F. Mak, and J. Shan, Valley magnetoelectricity in single-layer $\mathrm{MoS}_{2}$, Nat. Mater. 16, 887 (2017).
[54] T. Furukawa, Y. Shimokawa, K. Kobayashi, and T. Itou, Observation of current-induced bulk magnetization in elemental tellurium, Nat. Commun. 8, 954 (2017).

[55] J. Xia, Y. Maeno, P. T. Beyersdorf, M. M. Fejer, and A. Kapitulnik, High Resolution Polar Kerr Effect Measurements of $\mathrm{Sr}_{2} \mathrm{RuO}_{4}$ : Evidence for Broken Time-Reversal Symmetry in the Superconducting State, Phys. Rev. Lett. 97, 167002 (2006).

[56] N. J. Curro, Nuclear magnetic resonance in the heavy fermion superconductors, Rep. Prog. Phys. 72, 026502 (2009).

[57] W. Wernsdorfer, From micro- to nano-SQUIDs: Applications to nanomagnetism, Supercond. Sci. Technol. 22, 064013 (2009).

[58] G. Chen et al., Signatures of tunable superconductivity in a trilayer graphene moiré superlattice, Nature (London) 572, 215 (2019).

[59] K.-T. Tsai et al., Correlated insulating states and transport signature of superconductivity in twisted trilayer graphene moiré of moiré superlattices, arXiv:1912.03375.

[60] S. Xu et al., Tunable van Hove singularities and correlated states in twisted monolayer-bilayer graphene, Nat. Phys. 17, 619 (2021).

[61] J. M. Park et al., Tunable strongly coupled superconductivity in magic-angle twisted trilayer graphene, Nature (London) $\mathbf{5 9 0}$, 249 (2021).

[62] S. Moriyama et al., Observation of superconductivity in bilayer graphene/hexagonal boron nitride superlattices, arXiv:1901.09356.

[63] X. Liu et al., Tunable spin-polarized correlated states in twisted double bilayer graphene, Nature (London) 583, 221 (2020).

[64] C. Shen et al., Correlated states in twisted double bilayer graphene, Nat. Phys. 16, 520 (2020).

[65] H. Q. Yuan, D. F. Agterberg, N. Hayashi, P. Badica, D. Vandervelde, K. Togano, M. Sigrist, and M. B. Salamon, $S$ Wave Spin-Triplet Order in Superconductors without Inversion Symmetry: $\mathrm{Li}_{2} \mathrm{Pd}_{3} \mathrm{~B}$ and $\mathrm{Li}_{2} \mathrm{Pt}_{3} \mathrm{~B}$, Phys. Rev. Lett. 97, 017006 (2006).

[66] K. Togano, P. Badica, Y. Nakamori, S. Orimo, H. Takeya, and K. Hirata, Superconductivity in the Metal Rich Li-Pd-B Ternary Boride, Phys. Rev. Lett. 93, 247004 (2004).

[67] A. B. Karki et al., Structure and physical properties of the noncentrosymmetric superconductor $\mathrm{Mo}_{3} \mathrm{Al}_{2} \mathrm{C}$, Phys. Rev. B 82, 064512 (2010).

[68] E. M. Carnicom et al., $\mathrm{TaRh}_{2} \mathrm{~B}_{2}$ and $\mathrm{NbRh}_{2} \mathrm{~B}_{2}$ : Superconductors with a chiral noncentrosymmetric crystal structure, Sci. Adv. 4, 7969 (2018).

[69] G. Chang et al., Topological quantum properties of chiral crystals, Nat. Mater. 17, 978 (2018). 